\title{
A version of Krasnoselskii's compression-expansion fixed point theorem in cones for discontinuous operators with applications
}

\author{
Rubén Figueroa ${ }^{\dagger}$, Rodrigo López Pouso ${ }^{\dagger}$ and Jorge Rodríguez-López \\ $\dagger$ Departamento de Estatística, Análise Matemática e Optimización, Universidade de Santiago de Compostela, \\ 15782, Facultade de Matemáticas, Campus Vida, Santiago, Spain.
}

\begin{abstract}
We introduce a new fixed point theorem of Krasnoselskii type for discontinuous operators. As an application we use it to study the existence of positive solutions of a second-order differential problem with separated boundary conditions and discontinuous nonlinearities.

2010 MSC: 34A12; 34A36; 58J20.

Keywords and phrases: Krasnoselskii fixed point theorem; Positive solutions; Discontinuous differential equations.
\end{abstract}

\section{Introduction}

A classical problem $[9,10,12]$ is that of the existence of positive solutions for the differential equation

$$
u^{\prime \prime}(t)+g(t) f(u(t))=0, \quad(0<t<1),
$$

along with suitable boundary conditions (BCs).

This problem arises in the study of radial solutions in $\mathbb{R}^{n}, n \geq 2$ for the partial differential equation (PDE)

$$
\Delta v+h(\|x\|) f(v)=0, \quad x \in \mathbb{R}^{n},\|x\| \in\left[R_{1}, R_{2}\right],
$$

with the appropriate boundary conditions, see $[4,9,10]$.

Recently, in the paper [8], the authors study the existence of non trivial radial solutions for a system of PDEs of the previous type. First, they turn the former problem into a system of ordinary differential equations similar to (1.1).

The main novelty in this paper is that we will let $f$ to be discontinuous.

The classical compression-expansion fixed point theorem of Krasnoselskii (see [2] or [13]) is a wellknown tool of nonlinear analysis and it has proved very useful to deduce existence of solutions for 
nonlinear problems. Here we prove a generalization of that theorem which allows discontinuous operators. The idea is similar to that employed in [5, 11], where Schauder's fixed point theorem was extended. Then we return to problem (1.1) along with Sturm-Liouville BCs and we use our extension of Krasnoselskii's theorem to get a result about existence of positive solutions when $f$ is not necessarily continuous.

\section{Krasnoselskii's fixed point theorem for discontinuous operators}

In the sequel we need the following definitions. A closed and convex subset $K$ of a Banach space $(X,\|\cdot\|)$ is a cone if it satisfies the following conditions:

(i) if $x \in K$, then $\lambda x \in K$ for all $\lambda \geq 0$;

(ii) if $x \in K$ and $-x \in K$, then $x=0$.

A cone $K$ defines the partial order in $X$ given by $x \preceq y$ if and only if $y-x \in K$.

Let $U$ be a relatively open subset of $K$ and let $T: \bar{U} \subset K \rightarrow K$ be an operator, not necessarily continuous.

Definition 2.1 The closed-convex envelope of an operator $T: \bar{U} \longrightarrow K$ is the multivalued mapping $\mathbb{T}: \bar{U} \longrightarrow 2^{K}$ given by

$$
\mathbb{T} x=\bigcap_{\varepsilon>0} \overline{\operatorname{co}} T\left(\bar{B}_{\varepsilon}(x) \cap \bar{U}\right) \quad \text { for every } x \in \bar{U},
$$

where $\bar{B}_{\varepsilon}(x)$ denotes the closed ball centered at $x$ and radius $\varepsilon$, and $\overline{\mathrm{co}}$ means closed convex hull.

In other words, we say that $y \in \mathbb{T} x$ if for every $\varepsilon>0$ and every $\rho>0$ there exist $m \in \mathbb{N}$ and a finite family of vectors $x_{i} \in \bar{B}_{\varepsilon}(x) \cap \bar{U}$ and coefficients $\lambda_{i} \in[0,1](i=1,2, \ldots, m)$ such that $\sum \lambda_{i}=1$ and

$$
\left\|y-\sum_{i=1}^{m} \lambda_{i} T x_{i}\right\|<\rho .
$$

The previous definition was formulated for open subsets of a cone, but it works for arbitrary nonempty subsets of a Banach space (see [11]).

Closed-convex envelopes (cc-envelopes, for short) need not be upper semicontinuous (usc, for short), see [3, Example 1.2], unless some additional assumptions are imposed on $T$.

Proposition 2.2 Let $\mathbb{T}$ be the cc-envelope of an operator $T: \bar{U} \longrightarrow K$. The following properties are satisfied:

1. If $T$ maps bounded sets into relatively compact sets, then $\mathbb{T}$ assumes compact values and it is usc;

2. If $T \bar{U}$ is relatively compact, then $\mathbb{T} \bar{U}$ is relatively compact. 
Proof. Let $x \in \bar{U}$ be fixed and let us prove that $\mathbb{T} x$ is compact. We know that $\mathbb{T} x$ is closed, so it suffices to show that it is contained in a compact set. To do so, we note that

$$
\mathbb{T} x=\bigcap_{\varepsilon>0} \overline{\operatorname{co}} T\left(\bar{B}_{\varepsilon}(x) \cap \bar{U}\right) \subset \overline{\operatorname{co}} T\left(\bar{B}_{1}(x) \cap \bar{U}\right) \subset \overline{\operatorname{co}} \overline{T\left(\bar{B}_{1}(x) \cap \bar{U}\right)},
$$

and $\overline{\mathrm{co}} \overline{T\left(\bar{B}_{1}(x) \cap \bar{U}\right)}$ is compact because it is the closed convex hull of a compact subset of a Banach space; see [1, Theorem 5.35]. Hence $\mathbb{T} x$ is compact for every $x \in \bar{U}$, and this property allows us to check that $\mathbb{T}$ is usc by means of sequences, see [1, Theorem 17.20]: let $x_{n} \rightarrow x$ in $\bar{U}$ and let $y_{n} \in \mathbb{T} x_{n}$ for all $n \in \mathbb{N}$ be such that $y_{n} \rightarrow y$; we have to prove that $y \in \mathbb{T} x$. Let $\varepsilon>0$ be fixed and take $N \in \mathbb{N}$ such that $\bar{B}_{\varepsilon}\left(x_{n}\right) \subset \bar{B}_{2 \varepsilon}(x)$ for all $n \geq N$. Then we have $y_{n} \in \overline{\operatorname{co}} T\left(\bar{B}_{\varepsilon}\left(x_{n}\right) \cap \bar{U}\right) \subset \overline{\operatorname{co}} T\left(\bar{B}_{2 \varepsilon}(x) \cap \bar{U}\right)$ for all $n \geq N$, which implies that $y \in \overline{\operatorname{co}} T\left(\bar{B}_{2 \varepsilon}(x) \cap \bar{U}\right)$. Since $\varepsilon>0$ was arbitrary, we conclude that $y \in \mathbb{T} x$.

Arguments are similar for the second part of the proposition. For every $x \in \bar{U}$ and $\varepsilon>0$ we have

$$
\overline{\operatorname{co}} T\left(\bar{B}_{\varepsilon}(x) \cap \bar{U}\right) \subset \overline{\operatorname{co}} \overline{T \bar{U}}
$$

and therefore $\mathbb{T} x \subset \overline{\mathrm{co}} \overline{T \bar{U}}$ for all $x \in \bar{U}$. Hence, $\overline{\mathbb{T}} \bar{U}$ is compact because it is a closed subset of the compact set $\overline{\operatorname{co}} \overline{T \bar{U}}$.

Now we recall the fixed point theorem mentioned above (see [13, Theorem 13.D]).

Theorem 2.3 (Krasnoselskit) Let $r_{i} \leq R(i=1,2)$ be positive numbers with $r_{1} \neq r_{2}$ and let $T$ : $\bar{B}(0, R) \cap K \rightarrow K$ be a compact mapping. Suppose that

(a) $T x \nsucceq x$ for all $x \in K$ with $\|x\|=r_{1}$,

(b) $T x \npreceq x$ for all $x \in K$ with $\|x\|=r_{2}$.

Then $T$ has at least a fixed point $x \in K$ such that

$$
\min \left\{r_{1}, r_{2}\right\}<\|x\|<\max \left\{r_{1}, r_{2}\right\}
$$

In this section we introduce a generalization of the previous theorem which is based on the following idea: given a possibly discontinuous operator $T$, we build its cc-envelope $\mathbb{T}$ and we prove that it has fixed points by means of the version of Krasnoselskii fixed point theorem for multivalued mappings given by Fitzpatrick-Petryshyn [6]. Then we impose suitable conditions on $T$ which, roughly speaking, guarantee that fixed points of $\mathbb{T}$ are fixed point of $T$ too.

For completeness, we recall [6, Theorem 3.2].

TheOrem 2.4 Let $X$ be a Fréchet space with a cone $K \subset X$. Let d be a metric on $X$ and let $r_{1}, r_{2} \in$ $(0, \infty), r=\max \left\{r_{1}, r_{2}\right\}$ and $F: \bar{B}(0, r) \cap K \longrightarrow 2^{K}$ u.s.c. and condensing. Suppose there exists a continuous seminorm $p$ such that $(I-F)\left(\bar{B}\left(0, r_{1}\right) \cap K\right)$ is p-bounded. Moreover, suppose that $F$ satisfies:

1. there is some $w \in K$ with $p(w) \neq 0$ and such that $x \notin F(x)+$ tw for any $t>0$ and $x \in \partial_{K} B\left(0, r_{1}\right)$; 
2. $\lambda x \notin F(x)$ for any $\lambda>1$ and $x \in \partial_{K} B\left(0, r_{2}\right)$.

Then $F$ has a fixed point $x_{0}$ with $\min \left\{r_{1}, r_{2}\right\} \leq d\left(x_{0}, 0\right) \leq \max \left\{r_{1}, r_{2}\right\}$.

We are already in a position to introduce and prove the main results in this section, namely, two extensions of Krasnoselskii fixed point theorem for discontinuous operators.

TheOREM 2.5 Let $r_{i} \leq R(i=1,2)$ with $r_{1} \neq r_{2}$ positive numbers and $T: \bar{B}(0, R) \cap K \rightarrow K$ a mapping such that $T(\bar{B}(0, R) \cap K)$ is relatively compact and

$$
\{x\} \cap \mathbb{T} x \subset\{T x\} \quad \text { for all } x \in \bar{B}(0, R) \cap K,
$$

where $\mathbb{T}$ is the cc-envelope of $T$ as defined in (2.2).

Suppose that

(a) $\lambda x \notin \mathbb{T} x$ for all $x \in K$ with $\|x\|=r_{1}$ and all $\lambda \geq 1$,

(b) there exists $w \in K$ with $\|w\| \neq 0$ such that $x \notin \mathbb{T} x+\lambda w$ for all $\lambda \geq 0$ and all $x \in K$ with $\|x\|=r_{2}$.

Then $T$ has at least a fixed point $x \in K$ such that

$$
\min \left\{r_{1}, r_{2}\right\}<\|x\|<\max \left\{r_{1}, r_{2}\right\}
$$

Proof. Notice that the multivalued mapping $\mathbb{T}$ fulfills all the conditions in Theorem 2.4, so there exists a point $x$ such that $x \in \mathbb{T} x$ and

$$
\min \left\{r_{1}, r_{2}\right\}<\|x\|<\max \left\{r_{1}, r_{2}\right\}
$$

Moreover we deduce from (2.3) that $x=T x$ because $\{x\} \cap \mathbb{T} x=\{x\}$.

A second result leans on compression-expansion type conditions.

THEOREM 2.6 Let $r_{i} \leq R(i=1,2)$ with $r_{1} \neq r_{2}$ positive numbers and $T: \bar{B}(0, R) \cap K \rightarrow K$ a mapping such that $T(\bar{B}(0, R) \cap K)$ is relatively compact and fulfills condition (2.3).

Let $\mathbb{T}$ be the cc-envelope of $T$ and suppose that

(i) $y \nsucceq x$ for all $y \in \mathbb{T} x$ and all $x \in K$ with $\|x\|=r_{1}$,

(ii) $y \npreceq x$ for all $y \in \mathbb{T} x$ and all $x \in K$ with $\|x\|=r_{2}$.

Then $T$ has at least a fixed point $x \in K$ such that

$$
\min \left\{r_{1}, r_{2}\right\}<\|x\|<\max \left\{r_{1}, r_{2}\right\}
$$

Proof. It suffices to show that all the conditions in Theorem 2.5 are satisfied. First, we show that condition $(i)$ implies condition $(a)$ in Theorem 2.5. Let $x \in K$ be such that $\|x\|=r_{1}$ and let $\lambda \geq 1$; we have to prove that $\lambda x \notin \mathbb{T} x$. Reasoning by contradiction, we assume that $y=\lambda x \in \mathbb{T} x$. Then we have

$$
y-x=(\lambda-1) x \in K \quad \text { (because } \lambda-1 \geq 0)
$$

and this implies that $y \succeq x$, a contradiction with condition $(i)$. 
Now for condition $(b)$ in Theorem 2.5. Once again we use a contradiction argument: we assume that for every $w \in K$ such that $\|w\| \neq 0$ we can find $x \in \partial_{K} B\left(0, r_{2}\right)$ and $\lambda \geq 0$ such that $x \in \mathbb{T} x+\lambda w$, i.e., there exists $y \in \mathbb{T} x$ such that $x=y+\lambda w$. Hence, $x-y=\lambda w \in K$, a contradiction with (ii).

REMARK 2.7 Condition (2.3) is weaker than continuity, since if $T$ is continuous then $\mathbb{T} x=\{T x\}$, so (2.3) is trivially satisfied. In addition, it is not difficult to find discontinuous mappings that verify this condition as we show in our next section.

Neither Theorem 2.5 nor 2.6 remain true if we replace $\mathbb{T}$ by $T$ in the assumptions, as we show in the following example.

EXAmple 2.8 In $X=\mathbb{R}^{2}$ we consider the cone $K=\mathbb{R}_{+}^{2}=\left\{(x, y) \in \mathbb{R}^{2}: x, y \geq 0\right\}$.

Let $0<r<R$ and define a mapping $T: K \rightarrow K$ in polar coordinates as

$$
T(\rho, \theta)= \begin{cases}(0,0), & \text { if } \rho \neq r, \\ \left(r, \frac{\pi}{2}\right), & \text { if } \theta \in\left[0, \frac{\pi}{4}\right), \rho=r, \\ (r, 0), & \text { if } \theta \in\left[\frac{\pi}{4}, \frac{\pi}{2}\right], \rho=r .\end{cases}
$$

Note that $\mathbb{T} x=\{T x\}=\{(0,0)\}$ for all $x \in K$ such that $\|x\| \neq r$ because $T$ is continuous at those points. For points $x=(r, \theta)$, with $\theta \in[0, \pi / 2]$, we have three possibilities: if $\theta \in[0, \pi / 4)$, then $\mathbb{T} x$ is the segment with endpoints $(0,0)$ and $(r, \pi / 2)$; if $\theta \in(\pi / 4, \pi / 2]$, then $\mathbb{T} x$ is the segment with endpoints $(0,0)$ and $(r, 0)$; finally, $\mathbb{T}(r, \pi / 4)$ is the triangle with vertices $(0,0),(r, 0)$ and $(r, \pi / 2)$. Therefore,

$$
\{x\} \cap \mathbb{T} x \subset\{T x\} \quad \text { for all } x \in K .
$$

Moreover, conditions ( $i$ ) and (ii) in Theorem 2.6 are satisfied if we replace $\mathbb{T}$ by $T$ (and we take $r_{1}=R$ and $\left.r_{2}=r\right)$. However, $T$ has no fixed point in $\bar{B}(0, R) \backslash B(0, r)$.

\section{Application to Sturm-Liouville problems}

We consider the following generalization of equation (1.1) with separated BCs:

$$
\begin{aligned}
& u^{\prime \prime}(t)+g(t) f(t, u(t))=0 \quad(0<t<1) \\
& \alpha u(0)-\beta u^{\prime}(0)=0 \\
& \gamma u(1)+\delta u^{\prime}(1)=0
\end{aligned}
$$

where $\alpha, \beta, \gamma, \delta \geq 0$ and $\Gamma:=\gamma \beta+\alpha \gamma+\alpha \delta>0$.

The usual approach to this problem consists in turning it into a fixed point problem with the integral operator

$$
T u(t):=\int_{0}^{1} G(t, s) g(s) f(s, u(s)) d s,
$$

where $G$ is the Green's function associated to the differential problem.

Motivated by this situation, we study existence of fixed points of Hammerstein integral operators

$$
T u(t):=\int_{0}^{1} k(t, s) g(s) f(s, u(s)) d s,
$$


defined in a suitable space. Here we consider $\mathcal{C}([0,1])$, endowed with the usual supremum norm $\|u\|=$ $\max _{t \in[0,1]}|u(t)|$.

Fixed points of $T$ will be looked for in the cone

$$
K=\left\{u \in \mathcal{C}([0,1]): u \geq 0, \min _{t \in[a, b]} u(t) \geq c\|u\|\right\}
$$

where $[a, b] \subset[0,1]$ and $c \in(0,1]$. This cone was introduced by Guo and it was intensively employed in recent years, for example, see [7, 9, 12].

We suppose that the terms of the Hammerstein equation (3.5) satisfy the following hypotheses:

(H1) $f:[0,1] \times[0, \infty) \longrightarrow[0, \infty)$ is such that:

(a) Compositions $f(\cdot, u(\cdot))$ are measurable whenever $u \in \mathcal{C}([0,1])$; and

(b) For each $r>0$ there exists $R>0$ such that $f(t, u) \leq R$ for a.a. $t \in[0,1]$ and all $u \in[0, r]$.

(H2) $g$ measurable and $g(s) \geq 0$ almost everywhere.

(H3) $k:[0,1] \times[0,1] \rightarrow[0, \infty)$ is continuous.

(H4) There exists a measurable function $\Phi:[0,1] \rightarrow[0, \infty)$ satisfying

$$
\Phi g \in L^{1}(0,1) \text { and } \int_{a}^{b} \Phi(s) g(s) d s>0,
$$

and a constant $c \in(0,1]$ such that

$$
\begin{array}{ll}
k(t, s) \leq \Phi(s) & \text { for all } t, s \in[0,1] \\
c \Phi(s) \leq k(t, s) & \text { for all } t \in[a, b], s \in[0,1] .
\end{array}
$$

REMARK 3.1 Conditions $(H 1)-(H 4)$ are similar to those requested in [9] with the exception that we do not require $f$ to be continuous. In addition, our assumptions are more general than those in [10] or [12] where the authors require $g \in L^{1}(0,1)$ and $\Phi \in \mathcal{C}([0,1])$.

Lemma 3.2 If conditions $(H 1)-(H 4)$ are satisfied, then the operator $T: K \rightarrow K$ introduced in (3.5) is well-defined and maps bounded sets into relatively compact sets.

Proof. The operator $T$ maps $K$ into $K$. Indeed, we have

$$
\|T u\|=\max _{t \in[0,1]}\left\{\int_{0}^{1} k(t, s) g(s) f(s, u(s)) d s\right\} \leq \int_{0}^{1} \Phi(s) g(s) f(s, u(s)) d s .
$$

Moreover,

$$
\min _{t \in[a, b]}\{T u(t)\} \geq c \int_{0}^{1} \Phi(s) g(s) f(s, u(s)) d s .
$$

Hence, $T u \in K$ for every $u \in K$.

Now we prove that if $B \subset K$ is an arbitrary nonempty bounded set, then $T B$ is relatively compact. Let $r>0$ such that $u \in B$ implies $0 \leq u(t) \leq r$ for all $t \in[0,1]$, and let $R>0$ be the constant associated to $r>0$ by condition $(H 1)(b)$. Given $u \in B$, we have

$$
\int_{0}^{1} k(t, s) g(s) f(s, u(s)) d s \leq R \int_{0}^{1} \Phi(s) g(s) d s<\infty,
$$


so $T B$ is uniformly bounded. To see that $T B$ is equicontinuous, it suffices to show that for every $\tau \in[0,1]$ and $t_{n} \rightarrow \tau$, we have

$$
\lim _{t_{n} \rightarrow \tau} \int_{0}^{1}\left|k\left(t_{n}, s\right) g(s) f(s, u(s))-k(\tau, s) g(s) f(s, u(s))\right| d s=0 \quad \text { uniformly in } u \in B .
$$

To prove it, we note that for every $u \in B$ we have

$$
\left|k\left(t_{n}, s\right) g(s) f(s, u(s))-k(\tau, s) g(s) f(s, u(s))\right| \leq R g(s)\left|k\left(t_{n}, s\right)-k(\tau, s)\right|
$$

which tends to zero as $n$ tends to infinity for a.a. $s \in[0,1]$ because $k$ is continuous in $[0,1]$. Moreover,

$$
R g(s)\left|k\left(t_{n}, s\right)-k(\tau, s)\right| \leq 2 R \Phi(s) g(s) \quad \text { for all } n \in \mathbb{N}
$$

and $2 R \Phi g \in L^{1}(0,1)$, by $(H 4)$, so the dominated convergence theorem and (3.7) yield (3.6).

Moreover suppose that the discontinuities of $f$ allow the operator $T$ to satisfy the condition

$$
\{u\} \cap \mathbb{T} u \subset\{T u\} \quad \text { for all } u \in K \cap \mathbb{T} K,
$$

where $\mathbb{T}$ is the multivalued mapping associated to $T$ defined in (2.2). Examples of this type of nonlinearities $f$ can be looked up in $[5,11]$.

Lemma 3.3 Suppose that condition (3.8) holds and that

$\left(I_{\rho}^{1}\right)$ There exist $\rho>0$ and $\varepsilon>0$ such that $f^{\rho, \varepsilon}<m$, where

$$
f^{\rho, \varepsilon}:=\sup _{0 \leq t \leq 1,0 \leq u \leq \rho+\varepsilon}\left\{\frac{f(t, u)}{\rho}\right\} \quad \text { and } \quad \frac{1}{m}:=\sup _{t \in[0,1]} \int_{0}^{1} k(t, s) g(s) d s .
$$

Then $\lambda u \notin \mathbb{T} u$ for all $u \in \partial_{K} B(0, \rho)$ and all $\lambda \geq 1$.

Proof. Suppose that there exist $\lambda \geq 1$ and $u \in \partial_{K} B(0, \rho)$ such that $\lambda u=T v$ for some $v \in \bar{B}_{\varepsilon}(u) \cap K$, i.e.,

$$
\lambda u(t)=\int_{0}^{1} k(t, s) g(s) f(s, v(s)) d s .
$$

Taking the supremum for $t \in[0,1]$,

$$
\lambda \rho \leq \sup _{t \in[0,1]} \int_{0}^{1} k(t, s) g(s) f(s, v(s)) d s \leq \rho f^{\rho, \varepsilon} \sup _{t \in[0,1]} \int_{0}^{1} k(t, s) g(s) d s \leq \rho f^{\rho, \varepsilon} \frac{1}{m}<\rho,
$$

a contradiction.

Given $m \in \mathbb{N}$, it is similarly proved that $\lambda u \neq \sum_{i=1}^{m} \lambda_{i} T v_{i}$ for any $v_{i} \in \bar{B}_{\varepsilon}(u) \cap K$ and $\lambda_{i} \in[0,1]$ with $\sum_{i=1}^{m} \lambda_{i}=1$. Hence, $\lambda u \notin \operatorname{co}\left(T\left(\bar{B}_{\varepsilon}(u) \cap K\right)\right)$.

To see $\lambda u \notin \overline{\mathrm{co}}\left(T\left(\bar{B}_{\varepsilon}(u) \cap K\right)\right)$ we consider two cases: $\lambda=1$ and $\lambda>1$.

If $\lambda=1$, we have $u \notin \mathbb{T} u$ because $u \neq T u$ and $\{u\} \cap \mathbb{T} u \subset\{T u\}$.

If $\lambda>1$, we obtain from (3.9) that $\lambda \rho \leq \rho$, that in this case suppose a contradiction too. 
In the sequel we denote

$$
V_{\rho}=\left\{u \in K: \min _{a \leq t \leq b} u(t)<\rho\right\} .
$$

In addition, it is trivial to see that $B(0, \rho) \cap K \subset V_{\rho} \subset B(0, \rho / c) \cap K$, and $V_{\rho}$ is a relatively open subset of $K$ (since minimum function is continuous).

Lemma 3.4 Suppose that condition (3.8) holds and that

$\left(I_{\rho}^{0}\right)$ There exist $\rho>0$ and $\varepsilon>0$ such that $f_{\rho, \varepsilon}>M(a, b)$, where

$$
f_{\rho, \varepsilon}:=\inf _{a \leq t \leq b, c(\rho-\varepsilon) \leq u \leq \frac{\rho}{c}+\varepsilon}\left\{\frac{f(t, u)}{\rho}\right\} \quad \text { and } \quad \frac{1}{M(a, b)}:=\inf _{t \in[a, b]} \int_{a}^{b} k(t, s) g(s) d s .
$$

Then $u \notin \mathbb{T} u+\lambda e$ for all $u \in \partial V_{\rho}$, all $\lambda \geq 0$ and $e(t) \equiv 1$.

Proof. Suppose there exist $u \in \partial V_{\rho}$ and $\lambda \geq 0$ such that $u=T v+\lambda e$ for some $v \in \bar{B}_{\varepsilon}(u) \cap K$. Then

$$
u(t)=\int_{0}^{1} k(t, s) g(s) f(s, v(s)) d s+\lambda .
$$

Notice that $\|v\| \leq\|u\|+\varepsilon \leq \frac{\rho}{c}+\varepsilon$ and $\min _{t \in[a, b]} v(t) \geq c\|v\| \geq c(\|u\|-\varepsilon) \geq c(\rho-\varepsilon)$. Therefore, for $t \in[a, b]$

$$
u(t)=\int_{0}^{1} k(t, s) g(s) f(s, v(s)) d s+\lambda \geq \int_{a}^{b} k(t, s) g(s) f(s, v(s)) d s+\lambda \geq \rho f_{\rho, \varepsilon} \int_{a}^{b} k(t, s) g(s) d s+\lambda .
$$

Taking the infimum in $[a, b]$ we have

$$
\rho \geq \rho f_{\rho, \varepsilon} \inf _{t \in[a, b]} \int_{a}^{b} k(t, s) g(s) d s+\lambda>\rho+\lambda
$$

a contradiction because $\lambda \geq 0$.

Given $m \in \mathbb{N}$, it is similar to check that $u \neq \sum_{i=1}^{m} \lambda_{i} T v_{i}+\lambda e$ for any $v_{i} \in \bar{B}_{\varepsilon}(u)$ and $\lambda_{i} \in[0,1]$ $(i=1, \ldots, n)$ with $\sum_{i=1}^{m} \lambda_{i}=1$. Hence,

$$
u \notin \operatorname{co}\left(T\left(\bar{B}_{\varepsilon}(u) \cap K\right)\right)+\lambda e .
$$

If we consider two cases: $\lambda=0$ and $\lambda>0$, and we work in a similar way than in the previous lemma we obtain that $u \notin \mathbb{T} u+\lambda e$.

THEOREM 3.5 Under the hypothesis (H1)-(H4) and (3.8), the Hammerstein integral operator (3.5) has at least a positive fixed point in $K$ if either of the following conditions hold:

(a) There exist $\rho_{1}, \rho_{2} \in(0, \infty)$ with $\rho_{1} / c<\rho_{2}$ such that $\left(I_{\rho_{1}}^{0}\right)$ and $\left(I_{\rho_{2}}^{1}\right)$ hold.

(b) There exist $\rho_{1}, \rho_{2} \in(0, \infty)$ with $\rho_{1}<\rho_{2}$ such that $\left(I_{\rho_{1}}^{1}\right)$ and $\left(I_{\rho_{2}}^{0}\right)$ hold.

Proof. It is an immediately consequence of the generalization of Krasnoselskii's Theorem 2.5 together with both lemmas above: Lemma 3.3 and Lemma 3.4 . 
REMARK 3.6 Multiplicity results can be obtained combining previous conditions (see [9]).

Now we return to the differential BVP (3.4). We will say that $u$ is a solution of that problem if $u \in W^{2,1}([0,1])$ (i.e, if $u \in \mathcal{C}^{1}([0,1])$ and $u^{\prime} \in \mathrm{AC}([0,1])$, where $\mathrm{AC}([0,1])$ denote the absolutely continuous functions space defined in $[0,1])$ and satisfies (3.4).

The problem (3.4) was widely studied looking for positive solutions [4, 9]. However, the novelty here is to let function $f$ be discontinuous. In [4], the authors consider the problem with $g(t) f(t, u)=h(t, u(t))$ where $h$ is continuous and they use a norm compression-expansion theorem in order to guarantee the existence of solutions. On the other hand, in [9], Lan considers $f$ autonomous and continuous and weaker conditions about $g$, he even replaces the hypothesis integrable by measurable, but it is necessary that $\int_{0}^{1} \Phi(s) g(s) d s<\infty$. Here, as $f$ can be discontinuous, we will require $g \in L^{1}(0,1)$.

We can write the differential problem (3.4) as

$$
u(t)=\int_{0}^{1} G(t, s) g(s) f(s, u(s)) d s=: T u(t),
$$

where $G$ is the associated Green function, that in this case [9] is given by

$$
G(t, s)=\frac{1}{\Gamma} \begin{cases}(\gamma+\delta-\gamma t)(\beta+\alpha s), & \text { if } 0 \leq s \leq t \leq 1 \\ (\beta+\alpha t)(\gamma+\delta-\gamma s), & \text { if } 0 \leq t<s \leq 1\end{cases}
$$

and it is non negative.

As $G(t, s) \leq G(s, s)$ for all $s, t \in[0,1]$, it is possible to choose

$$
\Phi(s)=G(s, s)=\frac{1}{\Gamma}(\gamma+\delta-\gamma s)(\beta+\alpha s)
$$

Moreover we can choose $a, b$ and $c$ in the following way [9]:

(C1) $a, b \in[0,1]$ such that $-\beta / \alpha<a<b<1+\delta / \gamma$, where we consider $\beta / \alpha=\infty$ if $\alpha=0$ and $\delta / \gamma=\infty$ if $\gamma=0$.

(C2) $c=\min \{(\gamma+\delta-\gamma b) /(\gamma+\delta),(\beta+\alpha a) /(\alpha+\beta)\}$.

These choices guarantee that $c \Phi(s) \leq G(t, s)$ for $t \in[a, b]$ and $s \in[0,1]$.

We shall work, as before, in the cone

$$
K=\left\{u \in \mathcal{C}[0,1]: u \geq 0, \min _{t \in[a, b]} u(t) \geq c\|u\|\right\}
$$

We allow $f:[0,1] \times[0, \infty) \rightarrow[0, \infty)$ to have discontinuities over the graphs of the following curves.

Definition 3.7 We say that $\gamma:[r, s] \subset I=[0,1] \rightarrow[0, \infty), \gamma \in W^{2,1}([r, s])$, is an admissible discontinuity curve for the differential equation $u^{\prime \prime}=-g(t) f(t, u)$ if one of the following conditions holds:

(a) $\gamma^{\prime \prime}(t)=-g(t) f(t, \gamma(t))$ for a.e. $t \in[r, s]$ (then we say $\gamma$ is viable for the differential equation),

(b) There exist $\varepsilon>0$ and $\psi \in L^{1}(r, s), \psi(t)>0$ for a.e. $t \in[r, s]$ such that either

$$
\gamma^{\prime \prime}(t)+\psi(t)<-g(t) f(t, y) \text { for a.e. } t \in I \text { and all } y \in[\gamma(t)-\varepsilon, \gamma(t)+\varepsilon]
$$


or

$$
\gamma^{\prime \prime}(t)-\psi(t)>-g(t) f(t, y) \text { for a.e. } t \in I \text { and all } y \in[\gamma(t)-\varepsilon, \gamma(t)+\varepsilon] \text {. }
$$

In this case we say that $\gamma$ is inviable.

Working with admissible discontinuity curves involves some technicalities gathered in the next lemma and its subsequent corollaries whose proofs will be omitted because they can be found in [11].

Lemma 3.8 ([11, Lemma 4.1]) Let $a, b \in \mathbb{R}, a<b$, and let $g, h \in L^{1}(a, b), g \geq 0$ a.e., and $h>0$ a.e. in $(a, b)$.

For every measurable set $J \subset(a, b)$ with $m(J)>0$ there is a measurable set $J_{0} \subset J$ with $m\left(J \backslash J_{0}\right)=$ 0 such that for every $\tau_{0} \in J_{0}$ we have

$$
\lim _{t \rightarrow \tau_{0}^{+}} \frac{\int_{\left[\tau_{0}, t\right] \backslash J} g(s) d s}{\int_{\tau_{0}}^{t} h(s) d s}=0=\lim _{t \rightarrow \tau_{0}^{-}} \frac{\int_{\left[t, \tau_{0}\right] \backslash J} g(s) d s}{\int_{t}^{\tau_{0}} h(s) d s} .
$$

Corollary 3.9 ([11, Corollary 4.2]) Let $a, b \in \mathbb{R}, a<b$, and let $h \in L^{1}(a, b)$ be such that $h>0$ a.e. in $(a, b)$.

For every measurable set $J \subset(a, b)$ with $m(J)>0$ there is a measurable set $J_{0} \subset J$ with $m\left(J \backslash J_{0}\right)=$ 0 such that for all $\tau_{0} \in J_{0}$ we have

$$
\lim _{t \rightarrow \tau_{0}^{+}} \frac{\int_{\left[\tau_{0}, t\right] \cap J} h(s) d s}{\int_{\tau_{0}}^{t} h(s) d s}=1=\lim _{t \rightarrow \tau_{0}^{-}} \frac{\int_{\left[t, \tau_{0}\right] \cap J} h(s) d s}{\int_{t}^{\tau_{0}} h(s) d s} .
$$

Corollary 3.10 ([11, Corollary 4.3]) Let $a, b \in \mathbb{R}, a<b$, and let $f, f_{n}:[a, b] \longrightarrow \mathbb{R}$ be absolutely continuous functions on $[a, b](n \in \mathbb{N})$, such that $f_{n} \rightarrow f$ uniformly on $[a, b]$ and for a measurable set $A \subset[a, b]$ with $m(A)>0$ we have

$$
\lim _{n \rightarrow \infty} f_{n}^{\prime}(t)=g(t) \quad \text { for a.a. } t \in A .
$$

If there exists $M \in L^{1}(a, b)$ such that $\left|f^{\prime}(t)\right| \leq M(t)$ a.e. in $[a, b]$ and also $\left|f_{n}^{\prime}(t)\right| \leq M(t)$ a.e. in $[a, b](n \in \mathbb{N})$, then $f^{\prime}(t)=g(t)$ for a.a. $t \in A$.

We shall also need the following result.

Lemma 3.11 If $M \in L^{1}(0,1), M \geq 0$ almost everywhere, then the set

$$
Q=\left\{u \in \mathcal{C}^{1}([0,1]):\left|u^{\prime}(t)-u^{\prime}(s)\right| \leq \int_{s}^{t} M(r) d r \quad \text { whenever } 0 \leq s \leq t \leq 1\right\},
$$

is closed in $\mathcal{C}([0,1])$ with the maximum norm topology.

Moreover, if $u_{n} \in Q$ for all $n \in \mathbb{N}$ and $u_{n} \rightarrow u$ uniformly in $[0,1]$, then there exists a subsequence $\left\{u_{n_{k}}\right\}$ which tends to $u$ in the $\mathcal{C}^{1}$ norm.

Proof. Let $\left\{u_{n}\right\}$ be a sequence of elements of $Q$ which converges uniformly on $[0,1]$ to some function $u \in \mathcal{C}([0,1])$; we have to show that $u \in Q$ and a subsequence $\left\{u_{n_{k}}\right\}$ tends to $u$ in the $\mathcal{C}^{1}$ norm.

Since each $u_{n}$ is continuously differentiable, the Mean Value Theorem guarantees the existence of some $t_{n} \in(0,1)$ such that

$$
u_{n}^{\prime}\left(t_{n}\right)=u_{n}(1)-u_{n}(0) .
$$


This implies the existence of some $K>0$ such that $\left|u_{n}^{\prime}\left(t_{n}\right)\right| \leq K$ for all $n \in \mathbb{N}$, because $\left\{u_{n}\right\}$ is uniformly bounded in $[0,1]$. Hence, for every $n \in \mathbb{N}$ and every $t \in[0,1]$, we have

$$
\left|u_{n}^{\prime}(t)\right| \leq\left|u_{n}^{\prime}(t)-u_{n}^{\prime}\left(t_{n}\right)\right|+\left|u_{n}^{\prime}\left(t_{n}\right)\right| \leq \int_{0}^{1} M(s) d s+K,
$$

so $\left\{u_{n}\right\}$ is bounded in the $\mathcal{C}^{1}$ norm. Moreover, the definition of $Q$ implies that the sequence $\left\{u_{n}^{\prime}\right\}$ is equicontinuous in $[0,1]$, so the Ascoli-Arzelá Theorem ensures that some subsequence of $\left\{u_{n}\right\}$, say $\left\{u_{n_{k}}\right\}$, which converges in the $\mathcal{C}^{1}$ norm to some $v \in \mathcal{C}^{1}([0,1])$. As a result, $u=v$, so $u$ is continuously differentiable in $[0,1]$ and $\left\{u_{n_{k}}\right\}$ tends to $u$ in the $\mathcal{C}^{1}$ norm. In particular, $\left\{u_{n_{k}}^{\prime}\right\}$ tends to $u^{\prime}$ uniformly in $[0,1]$.

Moreover, for $s, t \in[0,1], s \leq t$, and all $k \in \mathbb{N}$, we have

$$
\left|u_{n_{k}}^{\prime}(t)-u_{n_{k}}^{\prime}(s)\right| \leq \int_{s}^{t} M(r) d r
$$

and going to the limit as $k$ tends to infinity we deduce that $\left|u^{\prime}(t)-u^{\prime}(s)\right| \leq \int_{s}^{t} M(r) d r$.

We are now ready for the proof of the main result in this section.

THEOREM 3.12 Suppose that $f$ and $g$ satisfy the following hypothesis:

i. $f:[0,1] \times[0, \infty) \longrightarrow[0, \infty)$ is such that:

(a) Compositions $f(\cdot, u(\cdot))$ are measurable whenever $u \in \mathcal{C}([0,1])$; and

(b) For each $r>0$ there exists $R>0$ such that $f(t, u) \leq R$ for a.a. $t \in[0,1]$ and all $u \in[0, r]$.

ii. There exist admissible discontinuity curves $\gamma_{n}: I_{n}=\left[a_{n}, b_{n}\right] \rightarrow[0, \infty), n \in \mathbb{N}$, such that for a.a. $t \in I$ the function $u \mapsto f(t, u)$ is continuous on $[0, \infty) \backslash \bigcup_{\left\{n: t \in I_{n}\right\}}\left\{\gamma_{n}(t)\right\}$.

iii. $g \in L^{1}(0,1)$ and $g(s) \geq 0$ almost everywhere with $\int_{a}^{b} g(s) d s>0$, where $a$ and $b$ are given in $(C 1)$. Moreover, assume that one of the following conditions hold:

(a) There exist $\rho_{1}, \rho_{2} \in(0, \infty)$ with $\rho_{1} / c<\rho_{2}$ such that $\left(I_{\rho_{1}}^{0}\right)$ and $\left(I_{\rho_{2}}^{1}\right)$ hold.

(b) There exist $\rho_{1}, \rho_{2} \in(0, \infty)$ with $\rho_{1}<\rho_{2}$ such that $\left(I_{\rho_{1}}^{1}\right)$ and $\left(I_{\rho_{2}}^{0}\right)$ hold.

Then the differential problem with separated BCs (3.4) has at least a positive solution $u \in W^{2,1}([0,1])$.

Proof. The operator $T: K \rightarrow K$ given by

$$
T u(t)=\int_{0}^{1} G(t, s) g(s) f(s, u(s)) d s
$$

is well defined and it maps bounded sets into relatively compact ones, as consequence of Lemma 3.2. In addition, as $G$ is the Green function associated to a second-order homogeneous differential problem, $T u \in W^{2,1}([0,1])$ for all $u \in K$. On the other hand, given $u \in B\left(0, \rho_{2} / c\right) \cap K=K_{2}$, we have $g(t) f(t, u(t)) \in L^{1}[0,1]$, and there exists $M(t) \in L^{1}[0,1]$ such that

$$
h(t, u):=g(t) f(t, u) \leq M(t) \text { for a.e. } t \in[0,1] \text { and all } u \in K_{2} .
$$


We consider the set

$$
Q=\left\{u \in \mathcal{C}^{1}([0,1]):\left|u^{\prime}(t)-u^{\prime}(s)\right| \leq \int_{s}^{t} M(r) d r \quad(s \leq t)\right\},
$$

which is closed in $\left(\mathcal{C}([0,1]),\|\cdot\|_{\infty}\right)$ by virtue of Lemma 3.11 .

Hence, since $T K_{2} \subset Q$ and $Q$ is a closed and convex subset of $\mathcal{C}([0,1])$, we have $\mathbb{T} K_{2} \subset Q$.

Now we will prove that

$$
\{u\} \cap \mathbb{T} u \subset\{T u\} \quad \text { for all } u \in K_{2} \cap \mathbb{T} K_{2} .
$$

To do so, we fix an arbitrary function $u \in K_{2} \cap Q$ and we consider three different cases.

Case $1-m\left(\left\{t \in I_{n}: u(t)=\gamma_{n}(t)\right\}\right)=0$ for all $n \in \mathbb{N}$. Let us prove that then $T$ is continuous at $u$.

The assumption implies that for a.a. $t \in I$ the mapping $h(t, \cdot)$ is continuous at $u(t)$. Hence if $u_{k} \rightarrow u$ in $K_{2} \cap Q$ then

$$
h\left(t, u_{k}(t)\right) \rightarrow h(t, u(t)) \quad \text { for a.a. } t \in I,
$$

which, along with (3.13), yield $T u_{k} \rightarrow T u$ in $\mathcal{C}(I)$.

Case $2-m\left(\left\{t \in I_{n}: u(t)=\gamma_{n}(t)\right\}\right)>0$ for some $n \in \mathbb{N}$ such that $\gamma_{n}$ is inviable. In this case we can prove that $u \notin \mathbb{T} u$.

First, we fix some notation. Let us assume that for some $n \in \mathbb{N}$ we have $m\left(\left\{t \in I_{n}: u(t)=\right.\right.$ $\left.\left.\gamma_{n}(t)\right\}\right)>0$ and there exist $\varepsilon>0$ and $\psi \in L^{1}\left(I_{n}\right), \psi(t)>0$ for a.a. $t \in I_{n}$, such that (3.12) holds with $\gamma$ replaced by $\gamma_{n}$. (The proof is similar if we assume (3.11) instead of (3.12), so we omit it.)

We denote $J=\left\{t \in I_{n}: u(t)=\gamma_{n}(t)\right\}$, and we deduce from Lemma 3.8 that there is a measurable set $J_{0} \subset J$ with $m\left(J_{0}\right)=m(J)>0$ such that for all $\tau_{0} \in J_{0}$ we have

$$
\lim _{t \rightarrow \tau_{0}^{+}} \frac{2 \int_{\left[\tau_{0}, t\right] \backslash J} M(s) d s}{(1 / 4) \int_{\tau_{0}}^{t} \psi(s) d s}=0=\lim _{t \rightarrow \tau_{0}^{-}} \frac{2 \int_{\left[t, \tau_{0}\right] \backslash J} M(s) d s}{(1 / 4) \int_{t}^{\tau_{0}} \psi(s) d s} .
$$

By Corollary 3.9 there exists $J_{1} \subset J_{0}$ with $m\left(J_{0} \backslash J_{1}\right)=0$ such that for all $\tau_{0} \in J_{1}$ we have

$$
\lim _{t \rightarrow \tau_{0}^{+}} \frac{\int_{\left[\tau_{0}, t\right] \cap J_{0}} \psi(s) d s}{\int_{\tau_{0}}^{t} \psi(s) d s}=1=\lim _{t \rightarrow \tau_{0}^{-}} \frac{\int_{\left[t, \tau_{0}\right] \cap J_{0}} \psi(s) d s}{\int_{t}^{\tau_{0}} \psi(s) d s} .
$$

Let us now fix a point $\tau_{0} \in J_{1}$. From (3.16) and (3.17) we deduce that there exist $t_{-}<\tilde{t}_{-}<\tau_{0}$ and $t_{+}>\tilde{t}_{+}>\tau_{0}, t_{ \pm}$sufficiently close to $\tau_{0}$ so that the following inequalities are satisfied for all $t \in\left[\tilde{t}_{+}, t_{+}\right]$:

$$
\begin{aligned}
2 & \int_{\left[\tau_{0}, t\right] \backslash J} M(s) d s<\frac{1}{4} \int_{\tau_{0}}^{t} \psi(s) d s, \\
\int_{\left[\tau_{0}, t\right] \cap J} \psi(s) d s \geq & \int_{\left[\tau_{0}, t\right] \cap J_{0}} \psi(s) d s>\frac{1}{2} \int_{\tau_{0}}^{t} \psi(s) d s,
\end{aligned}
$$

and for all $t \in\left[t_{-}, \tilde{t}_{-}\right]$:

$$
\begin{aligned}
2 \int_{\left[t, \tau_{0}\right] \backslash J} M(s) d s & <\frac{1}{4} \int_{t}^{\tau_{0}} \psi(s) d s, \\
\int_{\left[t, \tau_{0}\right] \cap J} \psi(s) d s & >\frac{1}{2} \int_{t}^{\tau_{0}} \psi(s) d s .
\end{aligned}
$$


Finally, we define a positive number

$$
\tilde{\rho}=\min \left\{\frac{1}{4} \int_{\tilde{t}_{-}}^{\tau_{0}} \psi(s) d s, \frac{1}{4} \int_{\tau_{0}}^{\tilde{t}_{+}} \psi(s) d s\right\}
$$

and we are now in a position to prove that $u \notin \mathbb{T} u$. It suffices to prove the following claim:

Claim - Let $\varepsilon>0$ be given by our assumptions over $\gamma_{n}$ and let $\rho=\frac{\tilde{\rho}}{2} \min \left\{\tilde{t}_{-}-t_{-}, t_{+}-\tilde{t}_{+}\right\}$be where $\tilde{\rho}$ is as in (3.22). For every finite family $u_{i} \in \bar{B}_{\varepsilon}(x) \cap K$ and $\lambda_{i} \in[0,1](i=1,2, \ldots, m)$, with $\sum \lambda_{i}=1$, we have $\left\|u-\sum \lambda_{i} T u_{i}\right\| \geq \rho$.

Let $u_{i}$ and $\lambda_{i}$ be as in the Claim and, for simplicity, denote $y=\sum \lambda_{i} T u_{i}$. For a.a. $t \in J=\{t \in$ $\left.I_{n}: u(t)=\gamma_{n}(t)\right\}$ we have

$$
y^{\prime \prime}(t)=\sum_{i=1}^{m} \lambda_{i}\left(T u_{i}\right)^{\prime \prime}(t)=-\sum_{i=1}^{m} \lambda_{i} h\left(t, u_{i}(t)\right)
$$

On the other hand, for every $i \in\{1,2, \ldots, m\}$ and every $t \in J$ we have

$$
\left|u_{i}(t)-\gamma_{n}(t)\right|=\left|u_{i}(t)-u(t)\right|<\varepsilon,
$$

and then the assumptions on $\gamma_{n}$ ensure that for a.a. $t \in J$ we have

$$
y^{\prime \prime}(t)=-\sum_{i=1}^{m} \lambda_{i} h\left(t, u_{i}(t)\right)<\sum_{i=1}^{m} \lambda_{i}\left(\gamma_{n}^{\prime \prime}(t)-\psi(t)\right)=u^{\prime \prime}(t)-\psi(t) .
$$

Now for $t \in\left[t_{-}, \tilde{t}_{-}\right]$we compute

$$
\begin{aligned}
y^{\prime}\left(\tau_{0}\right)-y^{\prime}(t)= & \int_{t}^{\tau_{0}} y^{\prime \prime}(s) d s=\int_{\left[t, \tau_{0}\right] \cap J} y^{\prime \prime}(s) d s+\int_{\left[t, \tau_{0}\right] \backslash J} y^{\prime \prime}(s) d s \\
< & \int_{\left[t, \tau_{0}\right] \cap J} u^{\prime \prime}(s) d s-\int_{\left[t, \tau_{0}\right] \cap J} \psi(s) d s \\
& \quad+\int_{\left[t, \tau_{0}\right] \backslash J} M(s) d s \quad(\text { by }(3.24),(3.23) \text { and }(3.13)) \\
= & u^{\prime}\left(\tau_{0}\right)-u^{\prime}(t)-\int_{\left[t, \tau_{0}\right] \backslash J} u^{\prime \prime}(s) d s-\int_{\left[t, \tau_{0}\right] \cap J} \psi(s) d s+\int_{\left[t, \tau_{0}\right] \backslash J} M(s) d s \\
\leq & u^{\prime}\left(\tau_{0}\right)-u^{\prime}(t)-\int_{\left[t, \tau_{0}\right] \cap J} \psi(s) d s+2 \int_{\left[t, \tau_{0}\right] \backslash J} M(s) d s \\
< & u^{\prime}\left(\tau_{0}\right)-u^{\prime}(t)-\frac{1}{4} \int_{t}^{\tau_{0}} \psi(s) d s \quad(\text { by }(3.20) \text { and }(3.21)),
\end{aligned}
$$

hence $y^{\prime}(t)-u^{\prime}(t) \geq \tilde{\rho}$ provided that $y^{\prime}\left(\tau_{0}\right) \geq u^{\prime}\left(\tau_{0}\right)$. Therefore, by integration we obtain

$$
y\left(\tilde{t}_{-}\right)-u\left(\tilde{t}_{-}\right)=y\left(t_{-}\right)-u\left(t_{-}\right)+\int_{t_{-}}^{\tilde{t}_{-}}\left(y^{\prime}(t)-u^{\prime}(t)\right) d t \geq y\left(t_{-}\right)-u\left(t_{-}\right)+\tilde{\rho}\left(\tilde{t}_{-}-t_{-}\right),
$$

so if $y\left(t_{-}\right)-u\left(t_{-}\right) \leq-\rho$, then $\|y-u\| \geq \rho$. Otherwise, if $y\left(t_{-}\right)-u\left(t_{-}\right)>-\rho$, then we have $y\left(\tilde{t}_{-}\right)-u\left(\tilde{t}_{-}\right)>\rho$ and thus $\|y-u\| \geq \rho$ too.

Similar computations in the interval $\left[\tilde{t}_{+}, t_{+}\right]$instead of $\left[t_{-}, \tilde{t}_{-}\right]$show that if $y^{\prime}\left(\tau_{0}\right) \leq u^{\prime}\left(\tau_{0}\right)$ then we have $u^{\prime}(t)-y^{\prime}(t) \geq \tilde{\rho}$ for all $t \in\left[\tilde{t}_{+}, t_{+}\right]$and this also implies $\|y-u\| \geq \rho$. The claim is proven.

Case $3-m\left(\left\{t \in I_{n}: u(t)=\gamma_{n}(t)\right\}\right)>0$ only for some of those $n \in \mathbb{N}$ such that $\gamma_{n}$ is viable. Let us prove that in this case the relation $u \in \mathbb{T} u$ implies $u=T u$. 
Let us consider the subsequence of all viable admissible discontinuity curves in the conditions of Case 3 , which we denote again by $\left\{\gamma_{n}\right\}_{n \in \mathbb{N}}$ to avoid overloading notation. We have $m\left(J_{n}\right)>0$ for all $n \in \mathbb{N}$, where

$$
J_{n}=\left\{t \in I_{n}: u(t)=\gamma_{n}(t)\right\}
$$

For each $n \in \mathbb{N}$ and for a.a. $t \in J_{n}$ we have

$$
u^{\prime \prime}(t)=\gamma_{n}^{\prime \prime}(t)=-h\left(t, \gamma_{n}(t)\right)=-h(t, u(t))
$$

and therefore $u^{\prime \prime}(t)=-h(t, u(t))$ a.e. in $J=\cup_{n \in \mathbb{N}} J_{n}$.

Now we assume that $u \in \mathbb{T} u$ and we prove that it implies that $u^{\prime \prime}(t)=-h(t, u(t))$ a.e. in $I \backslash J$, thus showing that $u=T u$.

Since $u \in \mathbb{T} u$ then for each $k \in \mathbb{N}$ we can guarantee that we can find functions $u_{k, i} \in \bar{B}_{1 / k}(u) \cap K_{2}$ and coefficients $\lambda_{k, i} \in[0,1](i=1,2, \ldots, m(k))$ such that $\sum \lambda_{k, i}=1$ and

$$
\left\|u-\sum_{i=1}^{m(k)} \lambda_{k, i} T u_{k, i}\right\|<\frac{1}{k}
$$

Let us denote $y_{k}=\sum_{i=1}^{m(k)} \lambda_{k, i} T u_{k, i}$, and notice that $y_{k} \rightarrow u$ uniformly in $I$ and $\left\|u_{k, i}-u\right\| \leq 1 / k$ for all $k \in \mathbb{N}$ and all $i \in\{1,2, \ldots, m(k)\}$.

For every $k \in \mathbb{N}$ we have $y_{k} \in Q$ as defined in (3.14), and therefore Lemma 3.11 guarantees that $u \in Q$ and, up to a subsequence, $y_{k} \rightarrow u$ in the $\mathcal{C}^{1}$ topology.

For a.a. $t \in I \backslash J$ we have that $h(t, \cdot)$ is continuous at $u(t)$, so for any $\varepsilon>0$ there is some $k_{0}=k_{0}(t) \in \mathbb{N}$ such that for all $k \in \mathbb{N}, k \geq k_{0}$, we have

$$
\left|h\left(t, u_{k, i}(t)\right)-h(t, u(t))\right|<\varepsilon \quad \text { for all } i \in\{1,2, \ldots, m(k)\}
$$

and therefore

$$
\left|y_{k}^{\prime \prime}(t)+h(t, u(t))\right| \leq \sum_{i=1}^{m(k)} \lambda_{k, i}\left|h\left(t, u_{k, i}(t)\right)-h(t, u(t))\right|<\varepsilon .
$$

Hence $y_{k}^{\prime \prime}(t) \rightarrow-h(t, u(t))$ for a.a. $t \in I \backslash J$, and then Corollary 3.10 guarantees that $u^{\prime \prime}(t)=-h(t, u(t))$ for a.a. $t \in I \backslash J$.

Therefore the proof of condition (3.15) is over and we conclude by means of Theorem 3.5.

REMARK 3.13 The differential problem (3.4) contains Dirichlet and Robin problems, so the previous result generalizes the existence results given in [10], because here we allow $f$ be discontinuous.

\section{Competing interests}

The authors declare that they have no competing interests. 


\section{Acknowledgements}

Rodrigo López Pouso was partially supported by Ministerio de Economía y Competitividad, Spain, and FEDER, Project MTM2013-43014-P, and Xunta de Galicia R2014/002 and GRC2015/004. Rubén

Figueroa and Jorge Rodríguez-López were partially supported by Xunta de Galicia, project EM2014/032.

\section{References}

[1] C. D. Aliprantis and K. C. Border, Infinite dimensional analysis. A hitchhiker's guide, 3rd Ed., Springer-Verlag (2006).

[2] H. Amann, Fixed point equations and nonlinear eigenvalue problems in ordered Banach spaces, SIAM Review, 184 (1976), 620-709.

[3] K. Deimling, Multivalued differential equations, Walter de Gruyter, Berlin (1992).

[4] L. H. Erbe, S. Hu and H. Wang, Multiple positive solutions of some boundary value problems, J. Math. Anal. Appl. 184 (1994), 640-648.

[5] R. Figueroa and G. Infante, A Schauder-type theorem for discontinuous operators with applications to second-order BVPs, Fixed Point Theory Appl., 2016, 2016:53.

[6] P. M. Fitzpatrick and W. V. Petryshyn, Fixed point theorems and the fixed point index for multivalued mappings in cones, J. London Math. Soc., 211 (1975), 75-85.

[7] G. Infante, A short course on positive solutions of systems of ODEs via fixed point index, arXiv:1306.4875 [math.CA].

[8] G. Infante and P. Pietramala, Nonzero radial solutions for a class of elliptic systems with nonlocal BCs on annular domains, Nonlinear Differential Equations and Applications, 22 (2015), 9791003.

[9] K. Q. Lan, Multiple positive solutions of semilinear differential equations with singularities, $J$. London Math. Soc., 632 (2001), 690-704.

[10] K. Q. Lan and J. R. L. Webb, Positive solutions of semilinear differential equations with singularities, J. Differential Equations, 148 (1998), 407-421.

[11] R. López Pouso, Schauder's fixed-point theorem: new applications and a new version for discontinuous operators, Bound. Value Probl. (2012) 2012:7.

[12] J. R. L. Webb, Positive solutions of some three point boundary value problems via fixed point index theory, Nonlinear Analysis, 47 (2001), 4319-4332.

[13] E. Zeidler, Nonlinear functional Analysis and its Applications I, Springer, New York (1986). 